\title{
Efficacy of lower-limb muscle training modalities in severely dyspnoeic individuals with COPD and quadriceps muscle weakness: results from the DICES trial
}

\author{
Maurice J H Sillen, ${ }^{1}$ Frits M E Franssen, ${ }^{1}$ Jeannet M L Delbressine, ${ }^{1}$ Anouk W Vaes, ${ }^{1}$ \\ Emiel F M Wouters, ${ }^{1,2}$ Martijn A Spruit ${ }^{1}$
}

- Additional material is published online only. To view please visit the journal online (http://dx.doi.org/10.1136/ thoraxjnl-2013-204388).

${ }^{1}$ Department of Research \& Education, CIRO+, Centre of Expertise for Chronic Organ Failure, Horn, The Netherlands ${ }^{2}$ Department of Respiratory Medicine, Maastricht University Medical Centre (MUMC+), Maastricht, The Netherlands

\section{Correspondence to} Maurice Sillen, Department of Research \& Education, $\mathrm{CIRO}+$ Centre of Expertise for Chronic Organ Failure, Hornerheide 1, Horn 6085 NM,

The Netherlands;

mauricesillen@ciro-horn.n

Received 21 August 2013 Revised 4 December 2013 Accepted 12 December 2013 Published Online First 7 January 2014

\section{SLinked}

http://dx.doi.org/10.1136/ thoraxjnl-2014-205345

\section{ABSTRACT}

Rationale Strength training and neuromuscular electrical stimulation (NMES) improve lower-limb muscle function in dyspnoeic individuals with chronic obstructive pulmonary disease (COPD). However, high-frequency NMES (HF-NMES) and strength training have never been compared head-to-head; and effects of low-frequency NMES (LF-NMES) have never been studied in COPD. Therefore, the optimal training modality to improve lower-limb muscle function, exercise performance and other patient-related outcomes in individuals with severe COPD remains unknown.

Objectives To study prospectively the efficacy of HFNMES $(75 \mathrm{~Hz})$, LF-NMES $(15 \mathrm{~Hz})$ or strength training in severely dyspnoeic individuals with COPD with quadriceps muscle weakness at baseline.

Methods 120 individuals with COPD (FEV $: 33 \pm 1 \%$ predicted, men: $52 \%$, age: $64.8 \pm 0.8$ years) were randomised to HF-NMES, LF-NMES or strength training as part of a comprehensive inpatient pulmonary rehabilitation programme. No treadmill walking or stationary cycling was provided.

Measurements and main results Groups were comparable at baseline. Quadriceps muscle strength increased after HF-NMES (+10.8 Newton-metre $(\mathrm{Nm}))$ or strength training $(+6.1 \mathrm{Nm}$; both $p<0.01)$, but not after LF-NMES (+1.4 Nm; $p=0.43)$. Quadriceps muscle endurance, exercise performance, lower-limb fat-free mass, exercise-induced symptoms of dyspnoea and fatigue improved significantly compared with baseline after HF-NMES, LF-NMES or strength training. The increase in quadriceps muscle strength and muscle endurance was greater after HF-NMES than after LFNMES.

Conclusions HF-NMES is equally effective as strength training in severely dyspnoeic individuals with COPD and muscle weakness in strengthening the quadriceps muscles and thus may be a good alternative in this particular group of patients. HF-NMES, LF-NMES and strength training were effective in improving exercise performance in severely dyspnoeic individuals with COPD and quadriceps weakness.

Trial registration NTR2322

\section{INTRODUCTION}

Lower-limb muscle dysfunction is a prominent extrapulmonary feature in individuals with moderate-to-very severe chronic obstructive pulmonary disease (COPD) ${ }^{1}$ It is related to exercise intolerance, increased healthcare use and mortality. ${ }^{1}$ Physical inactivity is most probably its main underlying cause. ${ }^{1}$ Therefore, exercise-based pulmonary rehabilitation should be part of the integrated care of individuals with COPD. ${ }^{2}$

Lower-limb muscle dysfunction occurs frequently in severely dyspnoeic individuals with COPD. ${ }^{3}$ Therefore, there is great interest in effective rehabilitative modalities which do not evoke severe dyspnoea, such as strength training or transcutaneous neuromuscular electrical stimulation (NMES). ${ }^{2}$ Indeed, the metabolic load on the impaired respiratory system is relatively low during strength training or NMES in individuals with COPD. ${ }^{45}$

Strength training or high-frequency NMES (HF-NMES; stimulation frequencies $\geq 50 \mathrm{~Hz}$ ) increases quadriceps muscle function, exercise capacity and health status as compared with a nonexercise control group or sham NMES in individuals with COPD, respectively. ${ }^{67}$ To date, a head-to-head comparison of strength training and HF-NMES has not been done in severely dyspnoeic individuals with COPD. Therefore, it remains unknown whether, and to what extent, these training modalities may have similar effects in individuals with COPD. A priori, no differences were expected between the groups.

Low-frequency NMES (LF-NMES, at $15 \mathrm{~Hz}$ ) of lower-limb muscles increased exercise capacity in individuals with chronic heart failure. ${ }^{8}$ To date, the effects of LF-NMES at $15 \mathrm{~Hz}$ have not been studied in individuals with COPD. However, it seems reasonable to hypothesise that quadriceps muscle endurance will improve to a greater extent after LF-NMES, ${ }^{8} 9$ while quadriceps muscle strength will improve to a greater extent after HF-NMES. ${ }^{10}$

The aim of the DICES (Dyspnoeic Individuals with COPD: Electrical stimulation or Strength training) trial was to study the efficacy of HF-NMES, LF-NMES or strength training in severely dyspnoeic individuals with COPD with quadriceps muscle weakness at baseline.

\section{METHODS}

Please see online supplementary data for all details.

\section{Participants}

Individuals with COPD referred for a pulmonary rehabilitation programme at the Centre of 
Expertise for Chronic Organ Failure (CIRO+) were screened for eligibility. ${ }^{11}$ Inclusion criteria were primary diagnosis of COPD; baseline modified Medical Research Council (MRC) dyspnoea grade 3 or 4 and quadriceps weakness (peak torque $\leq 80 \%$ predicted). ${ }^{12}$ Exclusion criteria were neuromuscular diseases; joint disorders in hip, leg and/or knee; metal implants in hip, leg and/or knee; cardiac pacemaker or internal cardiac defibrillator and/or outpatient pulmonary rehabilitation programme.

\section{Design and procedures}

A prospective, single-blind, randomised controlled trial was designed which was approved by the medical ethical committee of the Maastricht University Medical Centre+ (MEC 09-3-072). The DICES trial was registered at http://www.trialregister.nl (NTR2322) before enrolment of the first subject. All participants gave written informed consent to participate. Some baseline findings of the DICES trial have been published. ${ }^{13}$

\section{Interventions}

Participants were randomly assigned to HF-NMES, LF-NMES or strength training (8 weeks, twice a day, five times a week). All sessions were supervised by a physiotherapist. Symptom scores for dyspnoea, fatigue and muscle pain were assessed before and directly after each session. ${ }^{14}$ The DICES trial was part of a regular 8-week inpatient pulmonary rehabilitation programme, including also non-exercising components such as occupational therapy, relaxation therapy, exacerbation management strategies, educational sessions and psychosocial counselling. ${ }^{2}$ The interdisciplinary treatment was comparable among the groups. None of the participants underwent treadmill walking or stationary ergometry cycling.

\section{NMES protocols}

Quadriceps and calf muscles of both legs were stimulated electrically with a portable battery-operated electrical stimulator, using eight carbon-rubber electrodes (Tensmed S84, Enraf-Nonius, Rotterdam, the Netherlands) (see online supplementary figure E1). ${ }^{5}$ After a continuous 3-min warm-up at $5 \mathrm{~Hz}$, intensity was adjusted to individual toleration during each 18-min session. The frequencies used were $75 \mathrm{~Hz}$ (HF-NMES) or $15 \mathrm{~Hz}$ (LF-NMES). ${ }^{5}$

\section{Strength training}

Strength training consisted of bilateral leg extension and bilateral leg press exercises (Technogym SpA, Gambettola, Italy). ${ }^{15}$ Both exercises started at $70 \%$ of one-repetition maximum, four sets of eight repetitions for each exercise with at least 2 min of recovery between each set. The training load was set to increase by $5 \%$ every 2 weeks. ${ }^{15}$

\section{Outcomes}

Primary outcome

The primary outcome was the change in isokinetic quadriceps muscle function (ie, peak muscle strength and endurance) using a Biodex (Biodex System 4 Pro, Biodex Medical Systems, Inc, New York, USA). ${ }^{16}$ Participants performed 30 sequential volitional maximal contractions at an angular velocity of $90 \%$, while seated upright and with the hip joint in $90^{\circ}$ of flexion. Quadriceps muscle strength was defined as the highest peak torque (Newton-metre $(\mathrm{Nm})$ ). Quadriceps muscle endurance was defined as the total amount of delivered work (Joules (J)) during the set of 30 repetitions. ${ }^{16}$ To minimise learning effects, the measurement was performed twice at the initial assessment. Best values were used for analyses.

\section{Secondary outcomes}

Functional exercise performance was measured with the 6-min walk test $(6 \mathrm{MWT})$, including a practice walk at initial assessment. ${ }^{17}$ The best value was used for further analyses. The constant work-rate cycling endurance test (CWRT, expressed in seconds) was performed at $75 \%$ of the measured peak cycling work rate. ${ }^{18}$ Dyspnoea and fatigue were assessed before and after exercise tests and at isotime during the postintervention CWRT. Anxiety and depression were assessed using the Hospital Anxiety Depression Scale. ${ }^{19}$ Disease-specific health status was measured using the St George's Respiratory Questionnaire (SGRQ). ${ }^{20}$ The Canadian Occupational Performance Measure (COPM), a semistructured interview performed by an occupational therapist, was used to assess problematic activities of daily life (ADLs), ${ }^{21}$ and has been shown to be reliable in individuals with COPD. ${ }^{22}$ Whole-body dual-energy $\mathrm{X}$-ray absorptiometry scan was used to assess body mass index and fat-free mass index (FFMI). ${ }^{23} \mathrm{~A}$ modified MRC dyspnoea scale was used to assess dyspnoea. ${ }^{24}$ In addition, age, sex, height, weight, comorbidities, ${ }^{25}$ pulmonary function parameters and arterial blood gases were collected at the initial assessment. $^{13}$

\section{Sample size calculation, randomisation, blinding and statistics}

The DICES trial was powered to detect a significant difference in the change in maximal quadriceps strength between HF-NMES and LF-NMES of $9.2 \mathrm{~kg}$ on average. ${ }^{26}$ Each intervention group needed to have 36 individuals with COPD. Adjusting for drop out and withdrawals, we aimed for a minimum of 40 individuals in each intervention group.

A randomisation schedule was generated by the computer for participants with and without the use of long-term oxygen treatment; and with or without hospitalisation for a COPD exacerbation within $<3$ months of enrolment. The sequence was concealed. Analyses were performed using SPSS for Windows, V.17.0.1 (SPSS, Inc, Chicago, Illinois, USA). Differences within groups were analysed using paired tests or Wilcoxon signed rank test. Groups were compared using one-way analysis of variance, $\chi^{2}$ test, Fisher's exact test or Kruskal-Wallis one-way analysis of variance, as appropriate. The Bonferroni t test was used as post hoc test. Correlation analyses were done using Pearson's or Spearman's correlations. The level of significance was set at $\leq 0.05$.

\section{RESULTS}

\section{Participants' flow}

Between September 2010 and November 2012, 120 individuals with COPD were included in the DICES trial (HF-NMES: $\mathrm{n}=41$; LF-NMES: $\mathrm{n}=39$; strength training: $\mathrm{n}=40$ ). Ninety-one individuals $(75.8 \%)$ were followed up at 8 weeks. The main reasons for dropping out were severe health problems, including hospital admission. Dropout rates were similar among intervention groups (figure 1).

\section{Baseline characteristics}

Participants generally had severe to very severe COPD, a poor diffusing capacity, explicit quadriceps muscle weakness, very severe dyspnoea, a poor functional and peak exercise performance and a poor health status (table 1). Participants used $10 \pm 1$ types of medications (see online supplementary table E1) and 


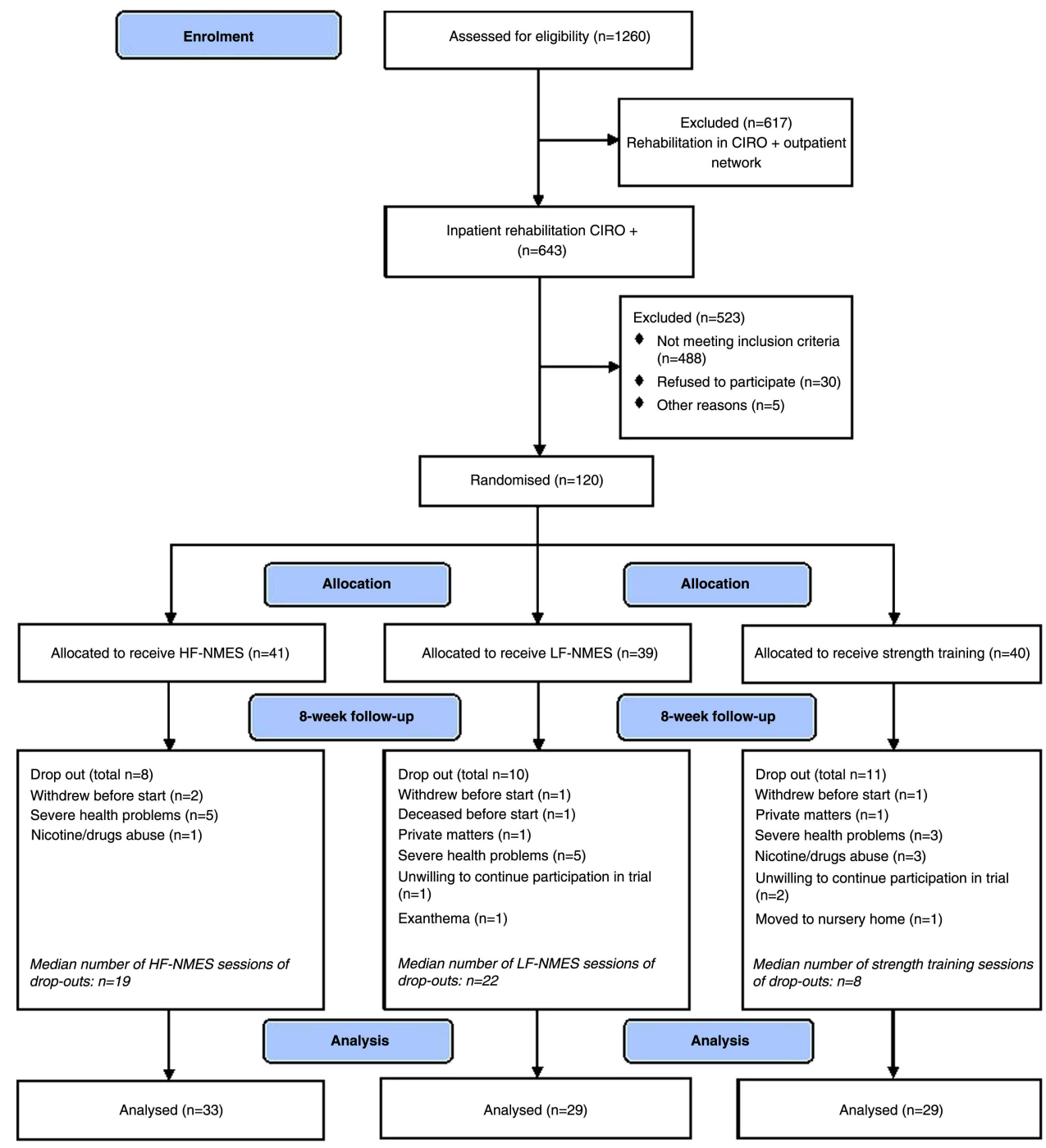

Figure 1 Flow diagram DICES trial (Dyspnoeic Individuals with COPD: Electrical stimulation or Strength training). CIRO, Centre of Expertise for Chronic Organ Failure; HF-NMES, high-frequency transcutaneous neuromuscular electrical stimulation; LF-NMES, low-frequency transcutaneous neuromuscular electrical stimulation.

had 3.2 \pm 0.1 objectified comorbidities (see online supplementary figure E2). The 29 individuals who dropped out and the 91 participants who completed the 8 -week intervention had similar baseline characteristics (see online supplementary table E2).

\section{Course of the 8-week NMES or strength training}

The 91 participants who completed the programme attended 76 \pm 3 HF-NMES sessions, 74 \pm 1 LF-NMES sessions, or $68 \pm 2$ strength training sessions (both NMES interventions vs strength training, $p<0.001)$. Exacerbations requiring antibiotics and/or corticosteroid treatment occurred frequently during all three interventions: $58 \%$ of the HF-NMES participants; $41 \%$ of the LF-NMES participants and $41 \%$ of the strength training participants $(p=0.254)$. During exacerbations of COPD which did not require hospital admission, NMES sessions (HF and LF) were continued. Strength training sessions were not always possible during exacerbations. The mean numbers of training sessions did not differ between the patients with and without one or more exacerbations $(p=0.564)$.

HF/LF-NMES current intensities and strength training loads increased significantly over time (figure 2). All training modalities were safe. No side effects, such as acute dyspnoea or muscle pain, were reported (see online supplementary figure E3 for all details).

\section{Efficacy}

Quadriceps muscle function

Isokinetic quadriceps peak torque increased significantly after HF-NMES $(10.8 \pm 2.9 \mathrm{Nm} ; \mathrm{p}<0.01)$ or strength training $(6.1$ $\pm 2.0 \mathrm{Nm}$; $\mathrm{p}<0.01)$, but not after LF-NMES $(1.4 \pm 1.8 \mathrm{Nm}$; $\mathrm{p}=0.43)$. Improvement in isokinetic quadriceps peak torque was significantly higher after HF-NMES compared with LF-NMES $(\mathrm{p}=0.01$; figure $3 \mathrm{~A})$. Isokinetic total work increased significantly in all groups (HF-NMES: $285 \pm 51 \mathrm{~J}$; LF-NMES: 101 $\pm 45 \mathrm{~J}$; strength training: $192 \pm 51 \mathrm{~J}$; all $\mathrm{p}<0.03$ ). Again, the improvement after HF-NMES was significantly higher than with LF-NMES ( $p=0.03$; figure 3B). Gains in peak muscle strength correlated with the increase in muscle endurance (HF-NMES: $r=0.689$; LF-NMES: $r=0.581$ and strength training: $r=0.689$, all $\mathrm{p}<0.001)$.

Six-minute walk test

Six-minute walk distance improved in all groups (HF-NMES: $66 \pm 14 \mathrm{~m}$; LF-NMES: $51 \pm 15 \mathrm{~m}$; strength training: $29 \pm 12 \mathrm{~m}$; 
Table 1 General characteristics

\begin{tabular}{|c|c|c|c|c|c|}
\hline Characteristics & $\begin{array}{l}\text { Total group } \\
(n=120)\end{array}$ & $\begin{array}{l}\text { HF-NMES } \\
(n=41)\end{array}$ & $\begin{array}{l}\text { LF-NMES } \\
(n=39)\end{array}$ & $\begin{array}{l}\text { Strength training } \\
(n=40)\end{array}$ & $\mathrm{p}$ Value \\
\hline $\operatorname{Sex}(M / F)$ & $62 / 58$ & $24 / 17$ & $19 / 20$ & $19 / 21$ & 0.555 \\
\hline Age (years) & $64.8 \pm 0.8$ & $64.4 \pm 1.3$ & $66.2 \pm 1.3$ & $64.0 \pm 1.3$ & 0.440 \\
\hline \multicolumn{6}{|l|}{ Pulmonary function } \\
\hline $\mathrm{FEV}_{1}(\mathrm{~L})$ & $0.85 \pm 0.03$ & $0.87 \pm 0.04$ & $0.87 \pm 0.07$ & $0.80 \pm 0.05$ & 0.578 \\
\hline $\mathrm{FEV}_{1}(\%$ predicted $)$ & $33 \pm 1$ & $33 \pm 2$ & $35 \pm 2$ & $33 \pm 2$ & 0.645 \\
\hline $\mathrm{FEV}_{1} / \mathrm{NC} \max (\%)$ & $32 \pm 1$ & $31 \pm 1$ & $31 \pm 2$ & $33 \pm 2$ & 0.545 \\
\hline Tlco (\% predicted) & $41 \pm 1$ & $39 \pm 2$ & $43 \pm 2$ & $42 \pm 3$ & 0.558 \\
\hline LTOT $(\%)$ & 51 & 56 & 54 & 43 & 0.429 \\
\hline GOLD classification (I/II/III/IV) & $0 / 12 / 36 / 72$ & $0 / 5 / 12 / 24$ & $0 / 2 / 14 / 23$ & $0 / 5 / 10 / 25$ & 0.942 \\
\hline GOLD classification (new) (A/B/C/D) & 0/3/0/117 & $0 / 2 / 0 / 39$ & 0/0/0/39 & 0/1/0/39 & 0.380 \\
\hline BMI $\left(\mathrm{kg} / \mathrm{m}^{2}\right)$ & $24.8 \pm 0.5$ & $24.1 \pm 0.8$ & $25.5 \pm 0.8$ & $24.9 \pm 0.8$ & 0.441 \\
\hline FFMI $\left(\mathrm{kg} / \mathrm{m}^{2}\right)$ & $16.5 \pm 0.2$ & $16.3 \pm 0.3$ & $16.6 \pm 0.3$ & $16.6 \pm 0.4$ & 0.688 \\
\hline Isokinetic quadriceps muscle function & $n=120$ & $\mathrm{n}=41$ & $\mathrm{n}=39$ & $\mathrm{n}=40$ & \\
\hline Peak torque $(\mathrm{Nm})$ & $76.2 \pm 2.4$ & $78.7 \pm 4.4$ & $76.1 \pm 4.1$ & $73.4 \pm 4.1$ & 0.682 \\
\hline Peak torque (\% predicted) & $54 \pm 1$ & $54 \pm 3$ & $55 \pm 2$ & $53 \pm 3$ & 0.812 \\
\hline Total work $(\mathrm{J})$ & $1175 \pm 44$ & $1189 \pm 87$ & $1164 \pm 67$ & $1175 \pm 76$ & 0.975 \\
\hline 6-Min walk test & $n=120$ & $n=41$ & $n=39$ & $\mathrm{n}=40$ & \\
\hline $6 \mathrm{MWD}(\mathrm{m})$ & $322 \pm 8$ & $311 \pm 16$ & $315 \pm 14$ & $337 \pm 14$ & 0.412 \\
\hline 6MWD (\% predicted) & $52 \pm 1$ & $48 \pm 3$ & $52 \pm 3$ & $54 \pm 3$ & 0.204 \\
\hline Cardiopulmonary exercise test & $n=104$ & $n=35$ & $n=33$ & $\mathrm{n}=36$ & \\
\hline Peak load $(W)$ & $44 \pm 1$ & $45 \pm 2$ & $45 \pm 2$ & $44 \pm 2$ & 0.984 \\
\hline Peak load (\% predicted) & $40 \pm 2$ & $33 \pm 3$ & $44 \pm 3$ & $44 \pm 5$ & 0.083 \\
\hline Peak $\mathrm{VO}_{2}(\mathrm{~mL} / \mathrm{min})$ & $820 \pm 21$ & $831 \pm 37$ & $829 \pm 43$ & $806 \pm 30$ & 0.858 \\
\hline Peak $\mathrm{VO}_{2}$ (\% predicted) & $58 \pm 5$ & $46 \pm 5$ & $57 \pm 6$ & $68 \pm 11$ & 0.179 \\
\hline Peak VE (\%MVV) & $94 \pm 4$ & $91 \pm 6$ & $93 \pm 6$ & $97 \pm 7$ & 0.790 \\
\hline Peak HR (\% predicted) & $75 \pm 1$ & $74 \pm 1$ & $75 \pm 3$ & $75 \pm 1$ & 0.830 \\
\hline Constant work-rate cycling endurance test & $\mathrm{n}=96$ & $n=33$ & $n=30$ & $n=33$ & \\
\hline Cycle time (s) & $191 \pm 10$ & $199 \pm 20$ & $188 \pm 15$ & $185 \pm 14$ & 0.836 \\
\hline Hospital Anxiety and Depression Scale & $\mathrm{n}=112$ & $n=39$ & $n=37$ & $\mathrm{n}=36$ & \\
\hline Anxiety (points) & $8.9 \pm 0.4$ & $7.3 \pm 0.7$ & $9.7 \pm 0.7$ & $9.8 \pm 0.6$ & 0.018 \\
\hline Depression (points) & $8.4 \pm 0.4$ & $8.1 \pm 0.5$ & $8.0 \pm 0.7$ & $9.1 \pm 0.7$ & 0.436 \\
\hline St George's Respiratory Questionnaire & $n=109$ & $\mathrm{n}=38$ & $n=36$ & $\mathrm{n}=35$ & \\
\hline Symptoms (points) & $66.3 \pm 1.6$ & $66.9 \pm 2.5$ & $67.0 \pm 2.9$ & $65.0 \pm 3.1$ & 0.850 \\
\hline Activity (points) & $81.7 \pm 1.6$ & $84.4 \pm 2.2$ & $80.9 \pm 3.0$ & $79.6 \pm 2.9$ & 0.431 \\
\hline Impact (points) & $53.1 \pm 1.7$ & $50.8 \pm 2.4$ & $52.8 \pm 3.4$ & $55.9 \pm 3.0$ & 0.462 \\
\hline Total score (points) & $63.9 \pm 1.3$ & $63.6 \pm 1.7$ & $63.7 \pm 2.6$ & $64.6 \pm 2.3$ & 0.932 \\
\hline
\end{tabular}

all $\mathrm{p}<0.03$; no differences between groups). Interestingly, symptoms at the end of the 6MWT were significantly lower than baseline after HF-NMES (dyspnoea: $6.9 \pm 0.4$ vs $5.7 \pm 0.4$ points; fatigue: $5.2 \pm 0.5$ vs $3.4 \pm 0.4$ points; both $\mathrm{p}<0.014$ ) or LF-NMES (dyspnoea: $6.4 \pm 0.4$ vs $5.4 \pm 0.5$ points; fatigue: 4.9 \pm 0.5 vs $2.8 \pm 0.5$ points; both $\mathrm{p}<0.032$ ). Symptoms at the end of the 6-min walk test remained unchanged after strength training (dyspnoea: $5.6 \pm 0.4$ vs $5.2 \pm 0.4$ points; fatigue: $4.1 \pm 0.5$ vs $3.3 \pm 0.5$ points; both $\mathrm{p}>0.11$ ).

\section{Constant work-rate test}

Endurance time during the CWRT improved in all groups (HF-NMES: $171 \pm 58 \mathrm{~s}$; LF-NMES: $167 \pm 46 \mathrm{~s}$; strength training: $69 \pm 30 \mathrm{~s}$; all $\mathrm{p}<0.03$; no differences between groups). At isotime, symptoms of dyspnoea were lower after both NMES protocols, and fatigue was lower after all three interventions compared with the initial CWRT (HF-NMES: $\Delta$ dyspnoea isotime: $-1.8 \pm 0.6$ points $(p=0.005), \Delta$ fatigue isotime: -2.6 \pm 0.6 points $(\mathrm{p}<0.001)$; LF-NMES: $-1.9 \pm 0.6$ points $(p=0.005)$ and $-1.4 \pm 0.5$ points $(p=0.01)$, respectively; and strength training: $0.4 \pm 0.4$ points $(p=0.361),-1.7 \pm 0.5$ points, $(p=0.003)$, respectively. Moreover, symptoms of fatigue at the end of the CWRT were significantly lower than at baseline after HF-NMES (6.3 \pm 0.5 vs $4.7 \pm 0.6$ points), LF-NMES $(5.8 \pm 0.5$ vs $5.0 \pm 0.6$ points) or strength training $(6.1 \pm 0.5$ vs $4.8 \pm 0.5$ points; all $\mathrm{p}<0.05)$. Symptoms of dyspnoea at the end of the cycle endurance test did not change (data not shown).

\section{Body composition}

Body mass index and FFMI did not change significantly compared with baseline in any of the groups. Lower-limb FFM increased in all groups (HF-NMES: $0.58 \pm 0.18 \mathrm{~kg}$; LF-NMES: 
A HF-NMES mm. quadriceps

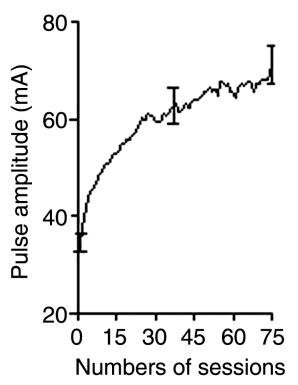

C LF-NMES mm. quadriceps

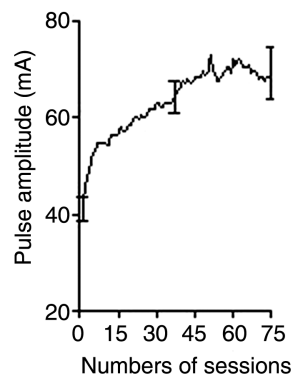

E Leg extension

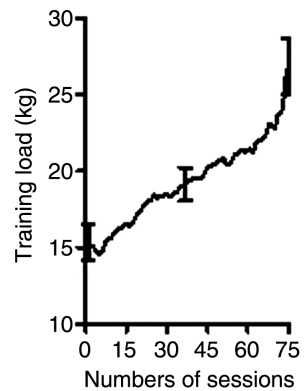

B HF-NMES mm. gastrocnemius

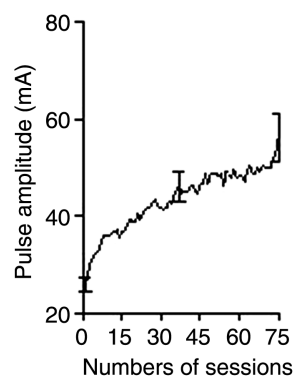

D LF-NMES mm. gastrocnemius

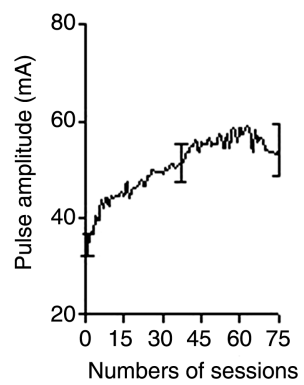

F Leg press

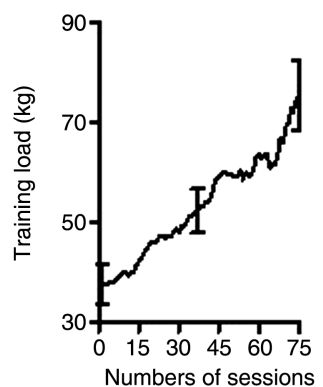

Figure 2 Course in NMES pulse amplitude and strength training load. Data are shown as mean \pm SEM. HF-NMES, high-frequency transcutaneous neuromuscular electrical stimulation; LF-NMES, low-frequency transcutaneous neuromuscular electrical stimulation.

$0.44 \pm 0.18 \mathrm{~kg}$; strength training: $0.37 \pm 0.13 \mathrm{~kg}$; all $\mathrm{p}<0.03$; no differences between groups).

\section{Dyspnoea}

Modified MRC dyspnoea scores improved in all groups (HF-NMES: $-0.9 \pm 0.2$ points; LF-NMES:

$-0.7 \pm 0.2$ points; strength training: $-0.8 \pm 0.2$ points; all $\mathrm{p}<0.005$; no differences between groups).

\section{Mood status}

Symptoms of depression improved in all groups (HF-NMES: $-1.8 \pm 0.6$ points; LF-NMES: $-2.2 \pm 0.5$ points; strength training: $-1.6 \pm 0.7$ points; all $\mathrm{p}<0.04)$, while symptoms of anxiety only improved after LF-NMES $(-1.8 \pm 0.6$ points; $\mathrm{p}=0.004)$. Changes in symptoms of anxiety or depression were similar between groups $(p>0.46)$.

\section{Health status}

Total SGRQ scores improved in all groups compared with baseline (HF-NMES: $-10.2 \pm 2.4$ points; LF-NMES: $-10.5 \pm 3.0$
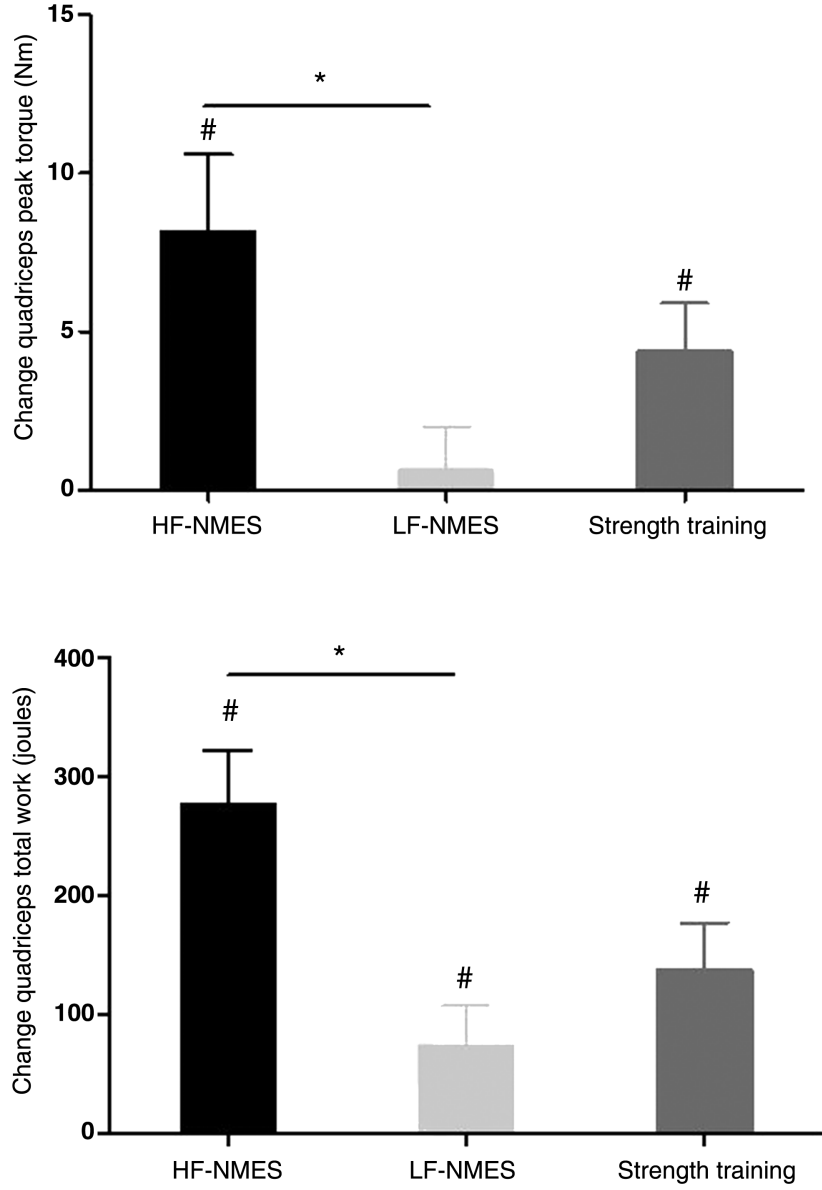

Figure 3 Change in quadriceps muscle strength and endurance. Data are shown as mean \pm SEM. ${ }^{*} p \leq 0.05 ; \# p \leq 0.05$ compared with baseline. HF-NMES, high-frequency transcutaneous neuromuscular electrical stimulation; LF-NMES, low-frequency transcutaneous neuromuscular electrical stimulation.

points; strength training: $-11.4 \pm 2.5$ points; all $\mathrm{p}<0.003$ ) Changes in health status scores were similar between groups $(p=0.948)$. See online supplementary table E5 for the significant changes in the SGRQ domain scores.

Problematic activities of daily living (ADL)

COPM total scores for problematic ADL improved in all groups compared with baseline (HF-NMES: performance: 2.7 \pm 0.2 points, satisfaction: $3.5 \pm 0.2$ points; LF-NMES: performance $2.5 \pm 0.2$ points, satisfaction $2.9 \pm 0.3$ points; strength training: performance $2.3 \pm 0.2$ points, satisfaction $2.8 \pm 0.3$ points; $\mathrm{p}<0.05$ ), with no significant differences between groups (see online supplementary tables E6 and E7 for details).

\section{DISCUSSION}

As far as we know, this is the first trial comparing the effects of HF-NMES, LF-NMES and strength training as the sole supervised muscle training modality during an 8-week pulmonary rehabilitation programme in severely dyspnoeic individuals with COPD and quadriceps weakness at baseline. Lower-limb muscle strength increased in the HF-NMES group and the strength training group compared with baseline. Exercise performance, exercise-induced symptoms of dyspnoea and fatigue improved significantly compared with baseline in all three lower-limb training modalities. Only the increase in isokinetic quadriceps 
muscle strength and endurance were higher after HF-NMES than after LF-NMES. Moreover, the DICES trial also showed that symptoms of depression, health status and problematic activities of daily living improved after an interdisciplinary pulmonary rehabilitation programme without treadmill walking or stationary cycling.

\section{Lower-limb muscle function}

Isokinetic quadriceps muscle strength improved after HF-NMES or strength training. This is in line with previous studies. ${ }^{6} 7$ Interestingly, no significant differences were found between HF-NMES and strength training. Therefore, HF-NMES may be a preferential muscle training modality in patients with COPD with severe dyspnoea and muscle weakness, as the metabolic load on the impaired cardiorespiratory system is significantly lower than with strength training. ${ }^{4}$ Moreover, HF-NMES recruits motor units in a non-selective, spatially fixed and temporally synchronous pattern, ${ }^{27}$ contrary to the activation order according to the size principle involving activation of the slower (lower force-producing) motor units before the faster (higher force-producing) units. $^{28}$ In COPD, atrophy of fast-twitch muscle fibres is consistently reported. ${ }^{29}$ It can be hypothesised that at least some of these fibres can be trained by HF-NMES, whereas these fibres might otherwise be activated only by highforce voluntary efforts. ${ }^{30}$

As expected from studies in healthy subjects, ${ }^{31}$ quadriceps muscle strength did not improve after LF-NMES. Indeed, the change in isokinetic quadriceps muscle strength was significantly higher in the HF-NMES group than in the LF-NMES group. Comparable results were found in a study comparing HF-NMES $(50 \mathrm{~Hz})$ with LF-NMES $(15 \mathrm{~Hz})$ in healthy volunteers and in individuals with chronic heart failure. ${ }^{10}$ The abovementioned results might be obtained because the increase in muscle strength is proportional to the stimulation frequency: higher frequencies produce higher torques, which probably result in a greater increase in muscle strength. ${ }^{32}$ Besides the peripheral pathway, HF-NMES can also produce muscle contractions by central recruitment through the electrically evoked sensory volley, in contrast to LF-NMES. ${ }^{33}$ During HF-NMES, the recruitment of motor units through central pathways can augment contractions generated through peripheral pathways, leading to the development of greater torques. ${ }^{34}$ While HF-NMES and LF-NMES had differential effects on muscle function in this study, the observed increase in lower-limb muscle mass was comparable, indicating that muscle dysfunction in COPD is not only related to the bulk of muscle.

\section{Exercise performance}

This trial shows that lower-limb muscle training modalities improve exercise performance significantly, while it is known that the load on the cardiorespiratory system is rather low. ${ }^{4}$ Indeed, the mean improvement in 6-min walk distance exceeded the minimal important differences of $25 \mathrm{~m}$ in all three intervention groups. ${ }^{35}$ Even though quadriceps muscle strength and endurance improved to a greater extent after HF-NMES than with LF-NMES, improvements in exercise performance were similar. This might be due to the fact that patients were still limited owing to their dyspnoea at the end of the exercise tests, while fatigue symptom scores were clearly lower at the end of the 6MWT or CWRT compared with baseline tests.

Dyspnoea, mood status, health status and problematic ADL Both strength training and HF-NMES have a positive impact on health status in individuals with COPD. ${ }^{7}$ Unfortunately, the design of our study does not allow us to distinguish between the impact of the muscle training modalities and the non-exercising parts of the pulmonary rehabilitation programme. The significant improvements in mood, health and problematic ADLs, however, were very encouraging as clinically relevant thresholds were exceeded, which is also true for walking and/or cyclingbased pulmonary rehabilitation programmes. ${ }^{2}$

\section{Strengths and methodological considerations}

The DICES trial has several strengths. This is the largest randomised controlled trial studying the efficacy of HF-NMES, LF-NMES or strength training in severely dyspnoeic individuals with COPD and a considerable number of comorbidities. The number of participants provided sufficient statistical power to detect possible differences in changes in isokinetic quadriceps muscle function between HF-NMES, LF-NMES and strength training. However, the DICES trial is probably underpowered to detect statistically significant differences between HF/LF-NMES and strength training for changes in exercise performance, which did exceed minimal important differences. Isokinetic quadriceps muscle function was assessed twice at the initial assessment to minimise a learning effect. Outcome assessors were blinded for group allocation. They were not (in)directly involved in the delivery of the interventions. This places greater credence on the results. ${ }^{36}$ Individuals randomly assigned to HF-NMES of LF-NMES were also blinded for the stimulation frequency applied. This is the first trial studying the efficacy of LF-NMES in individuals with COPD. All participants with baseline and outcome assessments were analysed, irrespective of the number of completed sessions or exacerbation treatment with antibiotics and/or corticosteroids during the intervention period.

The DICES trial also had some methodological limitations. The trial design did not include a control group, as strength training or HF-NMES had been proved to be beneficial compared with a non-exercise control group or sham NMES in individuals with COPD, respectively. ${ }^{67} 37$ Twenty-nine participants (24.2\%) did not complete the DICES trial. Dropping out appeared to be random across the DICES study sample as baseline characteristics were similar between participants who did and did not undergo outcome assessment (see online supplementary table E2). We believe that the drop-out rates are acceptable, as the DICES sample consisted of severely dyspnoeic individuals with COPD, who had muscle weakness and multiple coexisting morbidities. Moreover, our drop-out rate is comparable to drop-out rates in peer-reviewed COPD strength training trials (range: $11-38 \%$ ). ${ }^{6}$ The generalisability of our findings is limited owing to the strict inclusion and exclusion criteria. This approach, however, is in line with the philosophy of personalised medicine, in which healthcare is tailored to the individual patient or subgroups of patients. ${ }^{38}$

\section{CONCLUSIONS}

This study shows that HF-NMES is as effective as strength training in severely dyspnoeic individuals with COPD and quadriceps weakness in partially reversing quadriceps muscle dysfunction. HF-NMES may be a good alternative for strength training in this specific patient group. LF-NMES is not effective in improving muscle strength. HF-NMES, LF-NMES and strength training are effective training modalities, improving exercise performance, lower limb fat-free mass and health status in severely dyspnoeic individuals with COPD and quadriceps weakness. 
Acknowledgements The authors are grateful to the patients who volunteered for the DICES trial. We also thank Martijn Cuijpers and Martyna Renckens for their valuable help.

Contributors Trial concept and design: MJHS, EFMW and MAS; acquisition of data: MJHS, JMLD, AWV; analysis and interpretation of data, drafting the article: MJHS, FMEF and MAS; revising it critically for important intellectual content and final approval of the version to be published: all authors. MJHS had full access to al trial data and takes responsibility for the integrity of the data and the accuracy of the data analysis.

Funding Lung Foundation, Leusden, the Netherlands (grant 3.4.09.024); Weijerhorst Foundation, Maastricht, the Netherlands.

Ethics approval Medical ethical committee of the Maastricht University Medical Centre+, Maastricht, the Netherlands.

Provenance and peer review Not commissioned; externally peer reviewed.

\section{REFERENCES}

1 Maltais F, Decramer M, Barreiro E, et al. An official American Thoracic Society and European Respiratory Society statement; Update on Limb Muscle Dysfunction in COPD: 2013 update. Am J Respir Crit Care Med 2013: in press.

2 Spruit MA, Singh SJ, Garvey C, et al. An official American Thoracic Society/ European Respiratory Society statement: key concepts and advances in pulmonary rehabilitation. Am J Respir Crit Care Med 2013;188:e13-64.

3 Seymour JM, Spruit MA, Hopkinson NS, et al. The prevalence of quadriceps weakness in COPD and the relationship with disease severity. Eur Respir J 2010;36:81-8.

4 Sillen MJ, Janssen PP, Akkermans MA, et al. The metabolic response during resistance training and neuromuscular electrical stimulation (NMES) in patients with COPD, a pilot study. Respir Med 2008;102:786-9.

5 Sillen MJ, Wouters EF, Franssen FM, et al. Oxygen uptake, ventilation and symptoms during low-frequency versus high-frequency NMES in COPD: a pilot study. Lung 2011;189:21-6.

6 O'Shea SD, Taylor NF, Paratz JD. Progressive resistance exercise improves muscle strength and may improve elements of performance of daily activities for people with COPD: a systematic review. Chest 2009;136:1269-83.

7 Sillen MJ, Speksnijder CM, Eterman RM, et al. Effects of neuromuscular electrical stimulation of muscles of ambulation in patients with chronic heart failure or COPD: a systematic review of the English-language literature. Chest 2009;136:44-61.

8 Nuhr MJ, Pette D, Berger R, et al. Beneficial effects of chronic low-frequency stimulation of thigh muscles in patients with advanced chronic heart failure. Eur Heart J 2004;25:136-43.

9 Nuhr M, Crevenna R, Gohlsch B, et al. Functional and biochemical properties of chronically stimulated human skeletal muscle. Eur J Appl Physiol 2003;89:202-8.

10 Sbruzzi G, Schaan BD, Pimentel GL, et al. Effects of low frequency functional electrical stimulation with 15 and $50 \mathrm{~Hz}$ on muscle strength in heart failure patients. Disabil Rehabil 2011;33:486-93.

11 Spruit MA, Vanderhoven-Augustin I, Janssen PP, et al. Integration of pulmonary rehabilitation in COPD. Lancet 2008;371:12-13.

12 Borges 0 . Isometric and isokinetic knee extension and flexion torque in men and women aged 20-70. Scand I Rehabil Med 1989;21:45-53.

13 Sillen MJ, Franssen FM, Delbressine JM, et al. Heterogeneity in clinica characteristics and co-morbidities in dyspneic individuals with COPD GOLD D: findings of the DICES trial. Respir Med 2013;107:1186-94.

14 Revill SI, Robinson JO, Rosen M, et al. The reliability of a linear analogue for evaluating pain. Anaesthesia 1976;31:1191-8.

15 Spruit MA, Gosselink R, Troosters T, et al. Resistance versus endurance training in patients with COPD and peripheral muscle weakness. Eur Respir J 2002;19:1072-8.
16 Mathur S, Makrides L, Hernandez P. Test-retest reliability of isometric and isokinetic torque in patients with chronic obstructive pulmonary disease. Physiother Can 2004;56:94-101.

17 Hernandes NA, Wouters EF, Meijer K, et al. Reproducibility of 6-minute walking test in patients with COPD. Eur Respir J 2011:38:261-7.

18 Hul van 't A, Gosselink R, Kwakkel G. Constant-load cycle endurance performance: test-retest reliability and validity in patients with COPD. J Cardiopulm Rehabil 2003;23:143-50

19 Zigmond AS, Snaith RP. The hospital anxiety and depression scale. Acta Psychiatr Scand 1983:67:361-70.

20 Jones PW, Quirk FH, Baveystock CM. The St George's Respiratory Questionnaire. Respir Med 1991;85(Suppl B):25-31; discussion 3-7.

21 Annegarn J, Meijer K, Passos VL, et al. Problematic activities of daily life are weakly associated with clinical characteristics in COPD. J Am Med Dir Assoc 2012;13:284-90

22 Sewell L, Singh SJ. The Canadian occupational performance measure: is it a reliable measure in clients with chronic obstructive pulmonary disease? Br J Occup Ther 2001;64:305-10

23 Graat-Verboom L, Smeenk FW, van den Borne BE, et al. Progression of osteoporosis in patients with COPD: a 3-year follow up study. Respir Med 2012;106:861-70.

24 Spruit MA, Pennings HJ, Janssen PP, et al. Extra-pulmonary features in COPD patients entering rehabilitation after stratification for MRC dyspnea grade. Respir Med 2007;101:2454-63.

25 Vanfleteren LE, Spruit MA, Groenen M, et al. Clusters of comorbidities based on validated objective measurements and systemic inflammation in patients with chronic obstructive pulmonary disease. Am J Respir Crit Care Med 2013:187:728-35.

26 O'Shea SD, Taylor NF, Paratz JD. A predominantly home-based progressive resistance exercise program increases knee extensor strength in the short-term in people with chronic obstructive pulmonary disease: a randomised controlled trial. Aust J Physiother 2007;53:229-37.

27 Bickel CS, Gregory CM, Dean JC. Motor unit recruitment during neuromuscular electrical stimulation: a critical appraisal. Eur J Appl Physiol 2011;111:2399-407.

28 Henneman E, Somijen G, Carpenter DO. Functional significance of cell size in spinal motoneurons. J Neurophysiol 1965;28:560-80.

29 Gosker HR, Engelen MP, van Mameren H, et al. Muscle fiber type IIX atrophy is involved in the loss of fat-free mass in chronic obstructive pulmonary disease. Am J Clin Nutr 2002;76:113-19.

30 Maffiuletti NA. Physiological and methodological considerations for the use of neuromuscular electrical stimulation. Eur J Appl Physiol 2010;110:223-34.

31 Theriault R, Boulay MR, Theriault $G$, et al. Electrical stimulation-induced changes in performance and fiber type proportion of human knee extensor muscles. Eur J Appl Physiol Occup Physiol 1996;74:311-17.

32 Gregory CM, Dixon W, Bickel CS. Impact of varying pulse frequency and duration on muscle torque production and fatigue. Muscle Nerve 2007:35:504-9.

33 Collins DF. Central contributions to contractions evoked by tetanic neuromuscular electrical stimulation. Exerc Sport Sci Rev 2007;35:102-9.

34 Bergquist AJ, Clair JM, Lagerquist $\mathrm{O}$, et al. Neuromuscular electrical stimulation: implications of the electrically evoked sensory volley. Eur J Appl Physiol 2011:111:2409-26.

35 Holland AE, Hill CJ, Rasekaba T, et al. Updating the minimal important difference for six-minute walk distance in patients with chronic obstructive pulmonary disease. Arch Phys Med Rehabil 2010;91:221-5.

36 Schulz KF, Grimes DA. Blinding in randomised trials: hiding who got what. Lancet 2002;359:696-700.

37 Simpson K, Killian K, McCartney N, et al. Randomised controlled trial of weightlifting exercise in patients with chronic airflow limitation. Thorax. 1992:47:70-5.

38 WIKIPEDIA. 2013. http://en.wikipedia.org/wiki/Personalized_medicine (13 August 2013). 